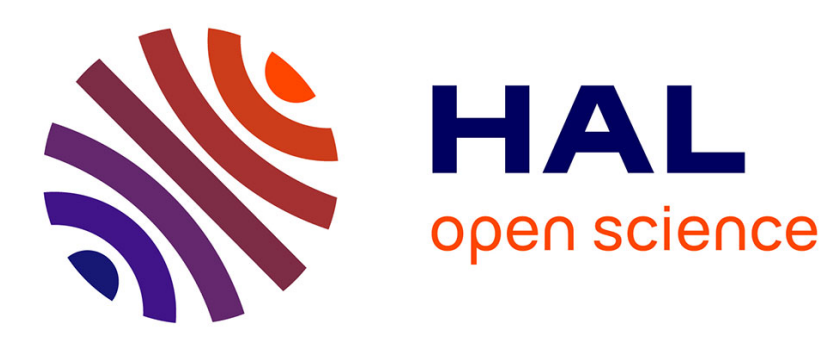

\title{
Identification-Based Approach for Electrical Coupling Compensation in a MEMS Gyroscope
}

Kévin Colin, Fabricio Saggin, Christophe Le Blanc, Xavier Bombois, Anton Korniienko, Gérard Scorletti

\section{- To cite this version:}

Kévin Colin, Fabricio Saggin, Christophe Le Blanc, Xavier Bombois, Anton Korniienko, et al.. Identification-Based Approach for Electrical Coupling Compensation in a MEMS Gyroscope. INERTIAL, Apr 2019, Naples, FL, United States. pp.1-4, 10.1109/ISISS.2019.8739573 . hal-02174925

\section{HAL Id: hal-02174925 \\ https://hal.science/hal-02174925}

Submitted on 26 May 2021

HAL is a multi-disciplinary open access archive for the deposit and dissemination of scientific research documents, whether they are published or not. The documents may come from teaching and research institutions in France or abroad, or from public or private research centers.
L'archive ouverte pluridisciplinaire HAL, est destinée au dépôt et à la diffusion de documents scientifiques de niveau recherche, publiés ou non, émanant des établissements d'enseignement et de recherche français ou étrangers, des laboratoires publics ou privés. 


\title{
Identification-Based Approach for Electrical Coupling Compensation in a MEMS Gyroscope
}

\author{
Kévin Colin ${ }^{1}$, Fabrício Saggin ${ }^{1}$, Christophe Le Blanc ${ }^{2}$, Xavier Bombois ${ }^{1}$, Anton Korniienko ${ }^{1}$, Gérard Scorletti ${ }^{1}$
}

\begin{abstract}
This work consists of a new approach to identify and compensate the parasitic electrical coupling between the excitation and the detection circuits of a MEMS gyroscope. Based on multivariable identification and the nonlinear behavior of electrostatic actuators, we propose a systematic and flexible way to model the mechanical modes as well as the parasitic coupling. The electrical coupling model is then used to compensate the parasitic effects of the device. Our main contributions are: (i) no model structure is enforced, so all the dynamics between actuation and detection are considered; and (ii) the multivariable framework allows also identifying the parasitic cross-couplings.
\end{abstract}

\section{INTRODUCTION}

In MEMS gyroscopes, the parasitic electrical coupling represents one of the major source of error for the angular rate measurement [1]. It is caused by a feed-through of the excitation circuits to the detection instrumentation [2]. To improve the performance of MEMS sensors, many papers are dedicated to the compensation of the parasitic electrical coupling, see, e.g., [2], [3], [4]. Among them, one can distinguish two types of compensation: analog and digital ones. The analog compensation is performed by implementing a compensation capacitance from the excitation to the detection circuit [3]. It allows to reduce the output power, avoiding the saturation of the detection circuit amplifiers. On the other hand, the digital compensation is realized by a digital filter [2], what makes this technique more flexible than the former one. Hence, we consider the digital compensation technique in this work.

The modeling of the parasitic electrical effect is required to design the compensation filter. In the literature, a common approach is followed: the model structure is enforced, and the model parameters are determined with experimental data. In [3], [4], the parasitic effect gain is constant near the resonance frequencies, justifying the use of a constant gain modeling. Nonetheless, the phase-delay is not well modeled, that is why in [2] a more complex model is chosen. This model corresponds to a feed-through of the inputs through a circuit made up by two capacitances and one resistor. The square nonlinearity is linearized for sinusoidal excitation thanks to the mechanical selective filtering around the resonance frequencies. This approach shows good results around the resonance frequencies but loses its validity on other frequencies due to the square nonlinearity linearization.

The model should be valid for a wider frequency range. Indeed, the sensor often operates in closed-loop. Then, the

\footnotetext{
${ }^{1}$ Laboratoire Ampere, UMR CNRS 5005, Ecole Centrale de Lyon, Université de Lyon, Ecully, France, kevin.colin@ec-lyon.fr

${ }^{2}$ Asygn, Grenoble, France, christophe. leblanceasygn.com
}

measurement noise passes through the feedback loop and can be amplified by the controller. This noise disturbs the output through the parasitic effect, degrading the measurement of the sensor. This motivates the development of a wider frequency range model without linearizing the square nonlinearity.

The parasitic effect cross-couples both mechanical resonant modes. In the literature, only the direct terms are considered [2]. Hence, in this paper, we develop a multivariable modeling of the mechanical transfer functions and the parasitic effect. Moreover, we consider a black-box approach associated to the Prediction Error method [5] for the model structure determination. By doing so, we capture all the parasitic effects in the model to compensate them. From this identified model, we design the digital compensation and verify its performances.

\section{DESCRIPTION OF THE MEMS GYROSCOPE AND PARASITIC ELECTRICAL COUPLING EFFECT}

\section{A. Description of the MEMS gyroscope}

The MEMS gyroscope is composed of two resonant masses, illustrated in Fig. 1. One mass $m_{x}$, attached to a fixed structure, oscillates along the $x$-axis. The other one $m_{y}$, attached to $m_{x}$, oscillates along the $y$-axis. The MEMS gyroscope is rotating around the $z$-axis with an angular rate $\Omega$. The resonant mode of $m_{x}$ (resp. $m_{y}$ ) is called the drive (resp. sense) mode and its resonance frequency is $f_{0_{x}}=11750 \mathrm{~Hz}$ (resp. $f_{0_{y}}=11868 \mathrm{~Hz}$ ) at $20^{\circ} \mathrm{C}$. This sensor uses the Coriolis effect for the angular rate deduction. The basis of its working principles are detailed in [6].

The drive (resp. sense) mass is actuated with an electrostatic force $f_{x}$ (resp. $f_{y}$ ) by using a comb-drive whose inbetween capacitance is proportional to the distance between the combs. By applying a voltage between them, an electrostatic force is generated, proportional to the square of this voltage. This input voltage on

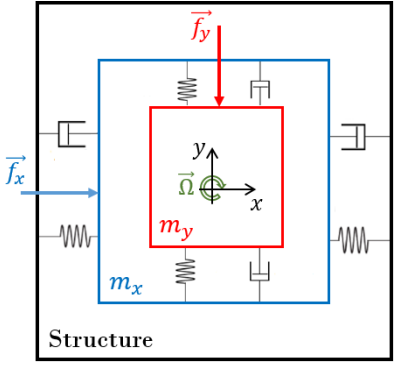

Fig. 1. MEMS gyroscope scheme. the drive (resp. sense) mode is denoted $v_{i n_{x}}$ (resp. $v_{i n_{y}}$ ). We measure the mass displacements $x$ and $y$ with combdrives, one per mass. In this case, each comb-drive is put in a detection circuit to convert the capacitance into an output voltage, still proportional to the corresponding mass displacement. The output voltage of the drive (resp. sense) mode is denoted $v_{\text {out }}$ (resp. $v_{\text {out }}$ ). 
The MEMS gyroscope is implemented on the MEMS development platform AS3125-SDK [7], which contains a multichannel high-performance reconfigurable IC front-end and a high-speed FPGA with microcontrollers, where identification and compensation routines are programmed. For the identification experiments, the signals are first sampled at $1 \mathrm{MHz}$ and then downsampled to a sampling frequency of $f_{s}=62500 \mathrm{~Hz}$. They are filtered through a first-order digital anti-aliasing filter with a cut-off frequency equal to $15 \mathrm{kHz}$.

\section{B. Parasitic electrical coupling and its compensation}

The use of comb-drives makes appear some nonidealities such as the parasitic electrical coupling effect. This effect can be modeled as a feed-through from the actuation to the detection circuit, depicted in Fig. 2 as the parasitic branch. This effect can be qualitatively seen in the direct transfer functions: an anti-resonance appears near the mechanical resonance for each mode [2] and the gains around the resonance are increased. This work aim to implement a multivariable digital filter that compensate the parasitic effects, as shown in Fig. 2.

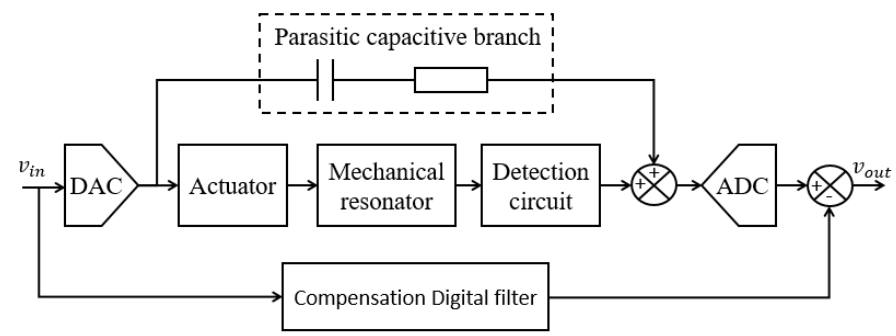

Fig. 2. Scheme of the digital compensation implementation.

\section{Modeling of THE MEMS Gyroscope}

\section{A. Description of the modeling approach}

We want to model and estimate the parasitic electrical coupling and the mechanical transfer. The parasitic effect is excited by the input voltages $v_{i n_{x}}$ and $v_{i n_{y}}$ while the mechanical transfer is excited by their square, i.e., $v_{i n_{x}}^{2}$ and $v_{i n_{y}}^{2}$. The idea of our approach is to identify all the transfers of both phenomena separately, i.e., to determine a multivariable model for each phenomenon with experimental data. For each phenomenon, we identify a discrete-time $2 \times 2$ transfer function matrix, denoted $\mathbf{E}$ for the electrical coupling and $\mathbf{G}$ for the mechanical transfer. The transfer from $v_{i n_{j}}$ to $v_{o u t_{i}}$ is denoted $E_{i j}$ and the one from $v_{i n_{j}}^{2}$ to $v_{o u t_{i}}$ is denoted $G_{i j}$ with $i, j \in\{x, y\}$. The model equations are given by:

$\left(\begin{array}{l}v_{\text {out }_{x}}(t) \\ v_{\text {out }_{y}}(t)\end{array}\right)=\mathbf{E}(z)\left(\begin{array}{l}v_{i n_{x}}(t) \\ v_{i n_{y}}(t)\end{array}\right)+\mathbf{G}(z)\left(\begin{array}{c}v_{i n_{x}}^{2}(t) \\ v_{i n_{y}}^{2}(t)\end{array}\right)+\left(\begin{array}{c}w_{x}(t) \\ w_{y}(t)\end{array}\right)$

where $z$ is the shift operator, $w_{x}(t)$ the measurement noise on the drive output, $w_{y}(t)$ the one on the sense output.

If we consider the voltages and their square as inputs, both phenomena are structurally separated. To simplify the modeling, we will identify each transfer function separately by exciting one input and measuring one output. The considered identification method is Prediction Error [5].

\section{B. Prediction Error Identification method}

Consider a linear system $\mathcal{S}$ expressed by

$$
\mathcal{S}: \quad \Upsilon(t)=F_{0}(z) u(t)+\nu(t)
$$

where $\Upsilon$ is the output, $u$ is the input, $F_{0}$ a stable and rational transfer function, and $\nu$ a noise process independent of $u$. We identify $F_{0}$ within a rational transfer function $F(z, \theta)$, where $\theta$ is the vector made up by the numerator and denominator coefficients of $F(z, \theta)$. The user can choose its numerator and denominator order. From $F(z, \theta)$, we define the predictor $\hat{\Upsilon}(t, \theta)$ and the prediction error $\epsilon(t, \theta)$ as

$$
\begin{aligned}
\hat{\Upsilon}(t, \theta) & =F(z, \theta) u(t) \\
\epsilon(t, \theta) & =\Upsilon(t)-\hat{\Upsilon}(t, \theta)=\Upsilon(t)-F(z, \theta) u(t)
\end{aligned}
$$

With $N$ input-output data generated from $\mathcal{S}$, we compute the optimal parameter vector $\theta$, denoted $\hat{\theta}_{N}$, minimizing a least-square cost-function on the prediction error:

$$
\hat{\theta}_{N}=\underset{\theta}{\operatorname{argmin}} \frac{1}{N} \sum_{t=1}^{N} \epsilon(t, \theta)^{2}
$$

One strong advantage of this method is the model verification tools: we can verify the chosen order of $F(z, \theta)$. The orders are well chosen when there is no significant correlation between $\epsilon\left(t, \hat{\theta}_{N}\right)$ and $u$ [5]. Another criterion, used in all modeling types, is the Best Fit. We generate other experimental data and compute the Best Fit expressed by:

$$
\text { Best Fit }=\left(1-\frac{\|\Upsilon-\hat{\Upsilon}\|_{2}}{\|\Upsilon\|_{2}}\right) \times 100 \%
$$

where $\|\cdot\|_{2}$ is the $\mathcal{L}_{2}$ norm. The closer to $100 \%$ the Best Fit is, the more accurate the model is.

\section{Experiment Design for the identification}

The inputs must be a multisine or a filtered white noise [5], imposed by the use of Prediction Error and designed such that mechanical transfer and parasitic effect can be separated.

By doing some preliminary experiments on the gyroscope, we know that the mechanical transfer is mainly located around the resonance frequencies $f_{0_{x}}$ and $f_{0_{y}}$. Therefore, for the modeling of $\mathbf{G}$, we should excite the resonances to maximize the signal-to-noise ratio (SNR). For the modeling of $\mathbf{E}$, as we want a wide frequency range model, we should excite it with a pseudo-random binary sequence (PRBS), whose spectrum is the same as a white noise process.

1) Experiment for the modeling of the mechanical transfer: we excite the gyroscope with multisine by choosing the excitation sinusoid frequencies around $f_{0_{x}} / 2$ and $f_{0_{y}} / 2$. The parasitic effect is then excited around $f_{0_{x}} / 2$ and $f_{0_{y}} / 2$ and the mechanical transfer is excited around $f_{0_{x}}$ and $f_{0_{y}}$, exciting the resonances. The mechanical effect can be modeled separately from the parasitic effect by filtering the output voltages to keep frequency range around $f_{0_{x}}$ and $f_{0_{y}}$.

For the experiments, the input voltage $v_{i n_{x}}$ (resp. $v_{i n_{y}}$ ) is composed of 100 sinusoids, in the frequency interval $[5850 \mathrm{~Hz}, 5900 \mathrm{~Hz}]($ resp. $[5910 \mathrm{~Hz}, 5960 \mathrm{~Hz}])$. For each transfer $G_{i j}$, after the experiment, we filter $v_{i n_{j}}^{2}$ and $v_{\text {out }}$ with a 
$10^{\text {th }}$ order band-pass Butterworth filter, keeping the double of the excited frequency range. Thus, the filtered output becomes $\Upsilon$ and the filtered input becomes $u$ for the identification.

2) Experiment for the modeling of the parasitic effect: we excite the gyroscope with a PRBS. To isolate the parasitic effect, the mechanical transfer effect is removed from the output. Hence, for the identification of $E_{i j}, \Upsilon$ is given by $\Upsilon(t)=v_{\text {out }_{i}}(t)-G_{i j}(z) v_{i n_{j}}^{2}(t)$ while $u$ is the PRBS $v_{i n_{j}}$.

\section{Identification results}

For each transfer function in $\mathbf{G}$ and $\mathbf{E}$, the number of data for the identification and the model verification is $N=50000$. First, thanks to the correlation analysis between $\epsilon\left(t, \hat{\theta}_{N}\right)$ and $u$, we have determined the right order to explain at best the experimental data for each transfer function. All the transfer functions in $\mathbf{G}$ are second order models and the ones in $\mathbf{E}$ are third order models. Contrary to [2], the first-order model is not enough to explain all the dynamics of the parasitic effect. One main reason is the anti-aliasing filter whose cutoff frequency is $15 \mathrm{kHz}$, near the resonance frequencies. The Best Fits for each transfer are given in Table I.

TABLE I

BEST FIT OF THE COMPUTED MODELS WITH THE VERIFICATION DATA.

\begin{tabular}{|c|c||c|c|}
\hline Model & Best Fit & Model & Best Fit \\
\hline$G_{x x}$ & $91.88 \%$ & $E_{x x}$ & $89.91 \%$ \\
\hline$G_{x y}$ & $50.31 \%$ & $E_{x y}$ & $94.13 \%$ \\
\hline$G_{y x}$ & $-1.24 \%$ & $E_{y x}$ & $34.23 \%$ \\
\hline$G_{y y}$ & $78.23 \%$ & $E_{y y}$ & $83.31 \%$ \\
\hline
\end{tabular}

The low Best Fit of $G_{y x}, G_{x y}$ and $E_{y x}$ are explained by a very low SNR. For $G_{y x}$, there is no visible effect in the data as it is strongly hidden by the measurement noise. It seems to be not relevant to model it and it will be carried out in future investigation. The Bode diagram magnitude of the transfer functions in $\mathbf{G}$ (except $G_{y x}$ ) are given in Fig. 3 and the ones in $\mathbf{E}$ are given in Fig. 4. The transfer functions in $\mathbf{E}$ have the same derivative effect in low frequencies $(+20 \mathrm{~dB} /$ decade slope) as explained in [2]. The gain reaches its maximum around the resonance frequencies $f_{0_{x}}$ and $f_{0_{y}}$. After, the gain decreases, due to the first order anti-aliasing filter.

\section{COMPENSATION OF THE PARASITIC EFFECT}

The considered compensation, whose implementation is illustrated in Fig. 2, is a $2 \times 2$ digital filter, as we want to compensate the four parasitic transfers. This multivariable filter is built by using the computed model of the parasitic electrical effect $\hat{\mathbf{E}}(z)=\mathbf{E}\left(z, \hat{\theta}_{N}\right)$. In this section, we evaluate the performances of the compensation. The Best Fits given in Table I are a first evaluation of this compensation. Indeed if the identified models in $\mathbf{E}$ represent perfectly the parasitic phenomena, then the filter compensates entirely the parasitic coupling. Here, the Best Fits are not equal to $100 \%$ and do not give full information on the modeling errors.

To verify the compensation, we excite the input voltage $v_{i n_{j}}$ with a PRBS oscillating between $0 \mathrm{~V}$ and $0.1 \mathrm{~V}$ and we measure the output voltage $v_{\text {out }}$ for all $i, j \in\{x, y\}$. Therefore, $v_{i n_{j}}^{2}$
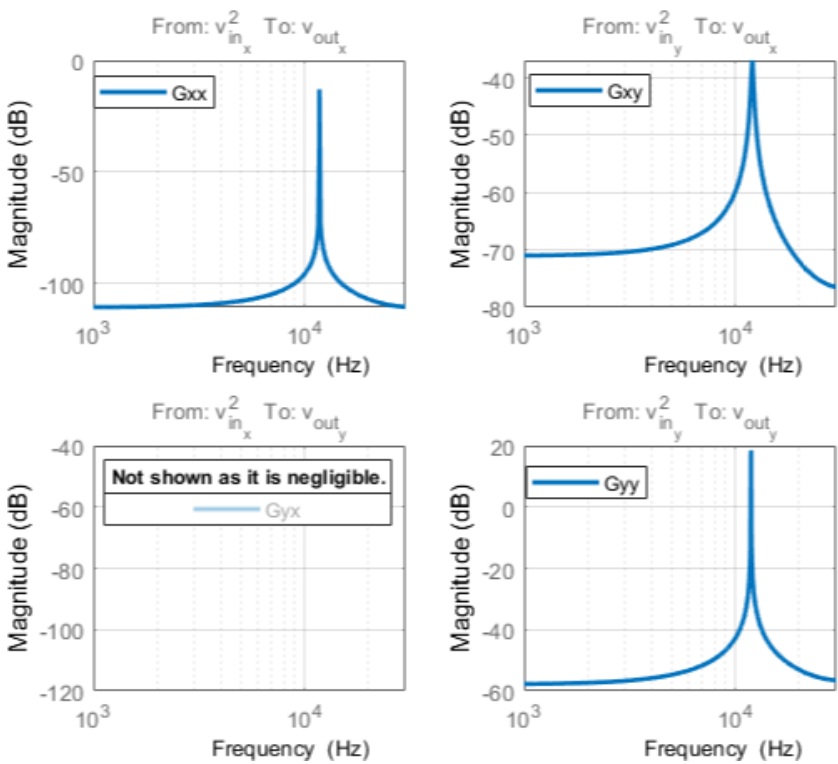

Fig. 3. Bode magnitude diagram of each transfer function in $\mathbf{G}(z)$.
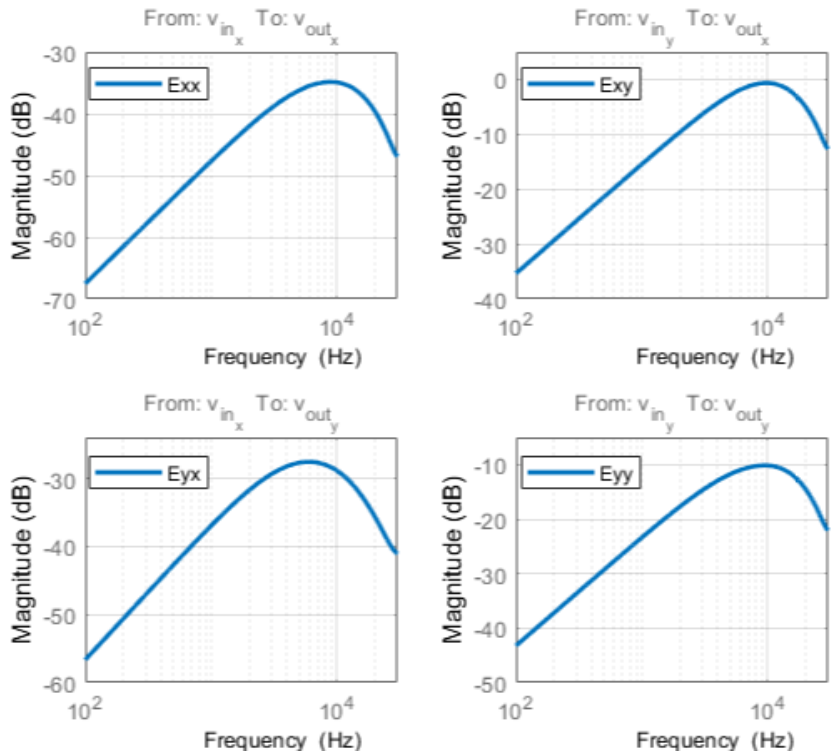

Fig. 4. Bode magnitude diagram of each transfer function in $\mathbf{E}(z)$.

is the same RBS, but oscillating between 0 and 0.01 . This choice of excitation is interesting since it allows to verify the compensation on the entire frequency range. To do so, we compute the periodogram of the output signal $v_{\text {out }}$ with and without compensation. We also compare both results with the output of the model $G_{i j}$ filtering $v_{i n_{j}}^{2}$. Due to lack of space in this paper, we only evaluate the compensation of the direct transfers. Fig. 5 presents the results of the drive direct transfer and Fig. 7 presents the results of the sense direct transfer. A zoom around the resonance frequencies is given respectively in Fig. 6 and Fig. 8. The experiments last 20s and the periodograms are smoothed with a span of 10 .

On both direct transfers, the anti-resonance due and the derivative effect are compensated. For the comparison with the output of the model $G_{x x}$ and $G_{y y}$, we have the same frequency 
behavior around the resonance frequencies. The difference at low and high frequencies are due to the measurement noise, not estimated in this work.

Finally, we quantify the power reduction of the digital signal. Table II gives the results on the output power with and without compensation, showing an important reduction of the power in both transfers.

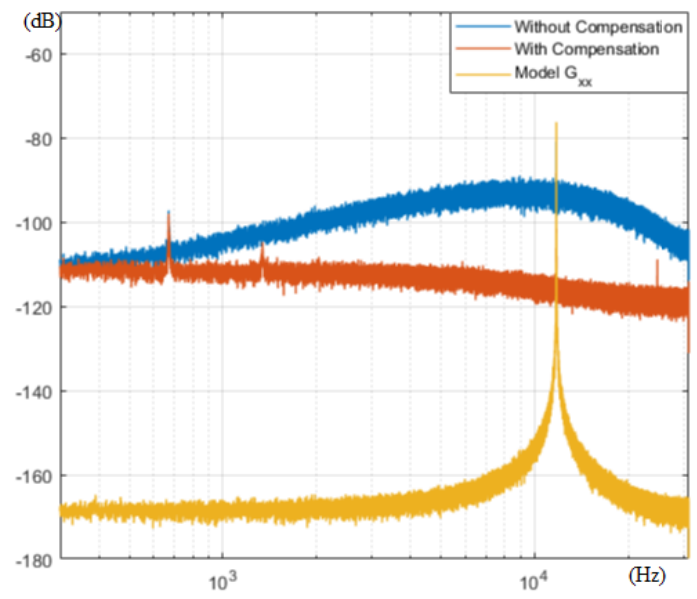

Fig. 5. Periodogram of $v_{o u t_{x}}$ for a PRBS in $v_{i n_{x}}$.

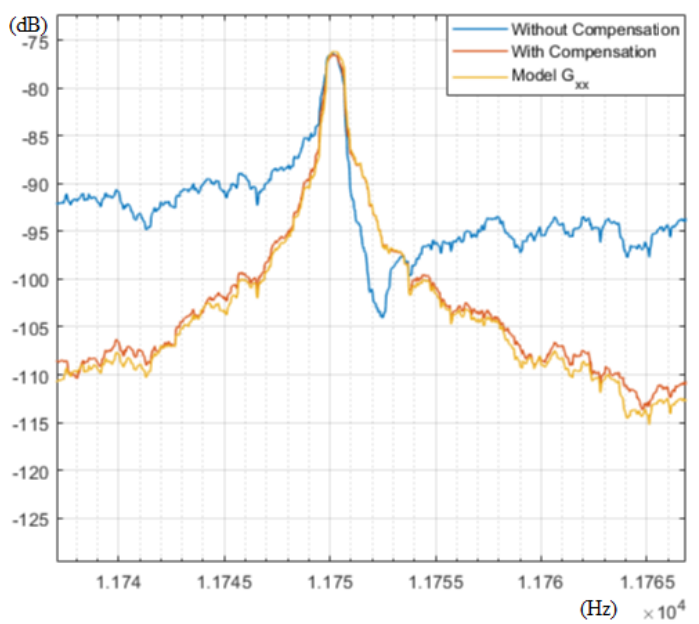

Fig. 6. Periodogram of $v_{\text {out }}$ for a PRBS in $v_{i n_{x}}$. Zoom around $f_{0_{x}}$.

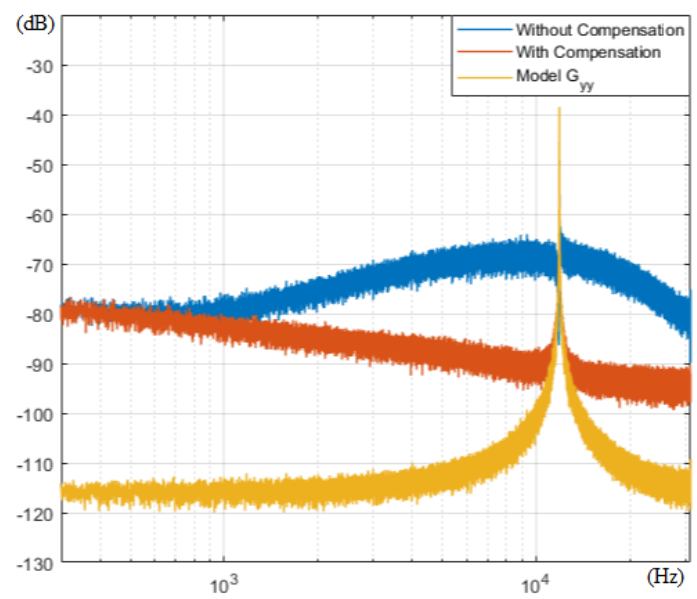

Fig. 7. Periodogram of $v_{o u t}$ for a PRBS in $v_{i n_{y}}$.

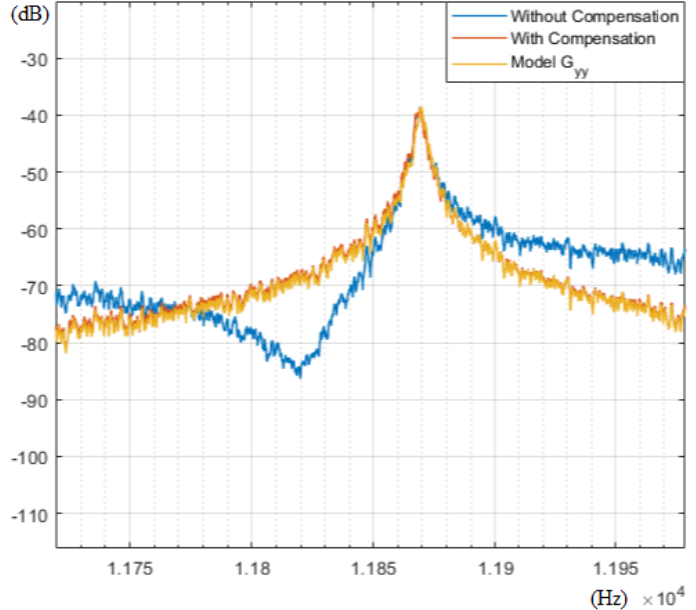

Fig. 8. Periodogram of $v_{o u t}$ for a PRBS in $v_{i n_{y}}$. Zoom around $f_{0_{y}}$.

TABLE II

OUTPUT POWER WITH AND WITHOUT COMPENSATION.

\begin{tabular}{|c|c|c|}
\hline Transfer & Drive to drive & Sense to sense \\
\hline Without compensation & $1.54 \times 10^{-4}$ & 0.0603 \\
\hline With compensation & $2.51 \times 10^{-6}$ & 0.0148 \\
\hline Relative power reduction & $98 \%$ & $75 \%$ \\
\hline
\end{tabular}

\section{CONClusion}

In this paper, we proposed a modeling method to separate the mechanical transfer and the parasitic electrical coupling without linearizing the actuation square nonlinearity. This allows a wider frequency range modeling. We use Prediction Error for the determination of the model complexity. This model is used as a compensation filter. The electrical coupling is successfully compensated in a wider frequency range. Furthermore, the Prediction Error method allows to study the model uncertainties of the compensation.

The approach presented in this work allows to compensate the coupling effects in the digital domain. However, since the coupling effects are also present in the analog domain, they can cause saturation on the detection circuit amplifiers. Thus, analog compensation strategies can be considered for a coarse compensation, and the digital technique can be used to perform a fine compensation.

\section{REFERENCES}

[1] M. J. Li, Y. P. Wang, Q. C. Zhao, C. H. He, L. T. Lin, J. F. Yang, and G. Z. Yan, "Study on the Influence Induced by Electrical Coupling Among Interconnection Lines in MEMS Gyroscopes," in 2017 19th Int. Conf. Solid-State Sensors, Actuators Microsystems. IEEE, jun 2017, pp. 1088-1091.

[2] C. Acar and A. M. Shkel, "An Approach for Increasing Drive-Mode Bandwidth of MEMS Vibratory Gyroscopes," J. Microelectromechanical Syst., vol. 14, no. 3, pp. 520-528, 2005.

[3] J. Giner and K. Ono, "Adaptive feedthrough cancellation in MEMS gyroscopes in reconfigurable IC+FPGA platform," in 2018 IEEE Int. Symp. Inert. Sensors Syst. IEEE, mar 2018, pp. 1-2.

[4] J. Raman, E. Cretu, P. Rombouts, and L. Weyten, "A Closed-Loop Digitally Controlled MEMS Gyroscope with Unconstrained Sigma-Delta Force-Feedback," IEEE Sens. J., vol. 9, no. 3, pp. 297-305, Mar. 2009.

[5] L. Ljung, System Identification: Theory for the User, 2nd ed. Pearson Education, 1998

[6] M. Saukoski, "System and Circuit Design for a Capacitive MEMS Gyroscope," Ph.D. dissertation, 2008.

[7] [Online]. Available: http://www.asygn.com/as3125-sdk 